\title{
COMPLEXITY AND INTERMITTENT TURBULENCE IN SPACE PLASMAS
}

Tom Chang and Sunny W.Y. Tam

Center for Space Research, Massachusetts Institute of Technology, Cambridge, MA 02139 USA

\section{Cheng-chin $\mathrm{Wu}$}

Department of Physics and Astronomy, University of California, Los Angeles, CA 90095 USA

Abstract Sporadic and localized interactions of coherent structures arising from plasma resonances can be the origin of "complexity" of the coexistence of nonpropagating spatiotemporal fluctuations and propagating modes in space plasmas. Numerical simulation results are presented to demonstrate the intermittent character of the non-propagating fluctuations. The technique of the dynamic renormalization-group is introduced and applied to the study of scale invariance of such type of multiscale fluctuations. We also demonstrate that the particle interactions with the intermittent turbulence can lead to the efficient energization of the plasma populations. An example related to the ion acceleration processes in the auroral zone is provided.

Key words Complexity, Space plasmas, Dynamic renormalization group, Forced and/or self-organized criticality, Topological phase transitions, Intermittency, Particle-Fluctuation interactions, Auroral ion acceleration. 


\section{Introduction}

In situ observations indicate that the dynamical processes in the space plasma environment generally entail anisotropic and localized intermittent fluctuations. It was suggested by Chang (1998a,b,c; 1999) that instead of considering this type of turbulence as an admixture of waves, such patchy intermittency could be more easily understood in terms of the development and interactions of coherent structures. Results of two-dimensional MHD simulations (Wu and Chang, 2000a,b; 2001) including the calculated fluctuation probability distribution functions and local intermittency measures (LIM) based on wavelet transforms seem to validate the suggested characteristics of the intermittent stochastic processes.

On the other hand, plasma waves are also generally observed in conjunction with the nonlinear, non-propagating fluctuations. It has been demonstrated that the coexistence of both propagating and non-propagating fluctuations in a plasma is a natural consequence of three-dimensional complexity for dynamical plasmas (Chang, 2003).

For nonlinear dynamical systems near criticality, the correlations among the fluctuations are extremely long-ranged. The dynamics of such systems are notoriously difficult to handle either analytically or numerically. . It has been suggested that the technique of the dynamic renormalization-group (Chang et al., 1992) might be capable of addressing such difficulties. Illustrative examples will be provided to demonstrate the utility of this technique in handling dynamical complexity of space plasmas.

It has been suggested that the intermittent non-propagating and propagating plasma fluctuations can interact efficiently with the charged plasma particles (Chang, 2001; Chang et al., 2003; 2004). This idea will be applied to the energization of ionospheric ions to magnetospheric energies in the auroral zone.

\section{Plasma Resonances and Coherent Structures}

Most field theoretical discussions begin with the concept of propagation of waves. For example, in the MHD formulation, one can combine the basic equations and express them in the following propagation forms:

$$
\rho d \mathbf{V} / d t=\mathbf{B} \cdot \nabla \mathbf{B}+\cdots, \quad d \mathrm{~B} / d t=\mathbf{B} \cdot \nabla \mathbf{V}+\cdots
$$

where the ellipses represent the effects of the anisotropic pressure tensor, the compressible and dissipative effects, and all notations are standard. 
PLASMAS

Equations (1.1) admit the well-known Alfvén waves. For such waves to propagate, the propagation vector $\mathbf{k}$ must contain a field-aligned component, i.e., $\mathbf{B} \cdot \nabla \rightarrow i \mathbf{k} \cdot \mathbf{B} \neq 0$. However, at sites where the parallel component of the propagation vector vanishes (i.e., at the resonance sites), the fluctuations are localized. Around these resonance sites (usually in the form of curves), it may be shown that the fluctuations are held back by the background magnetic field, forming Alfvénic coherent structures (Waddell et al., 1979; Tetreault, 1992a; Chang, 1998a.b.c; 1999; 2001; 2003, Chang et al., 2003).

Coarse-Grained Helicity. Let us now consider the geometry of the Alfvénic coherent structures. For an ideal MHD system, it has been suggested by Taylor (1974) that in a relaxed state such a structure would be approximately force-free (i.e., $\mathbf{J} \times \mathbf{B}=0$ ) due to the approximate conservation of the coarse-grained helicity defined as $K=\int \mathbf{A} \cdot \mathbf{B} d V$ integrated over the coherent structure, where $\mathbf{J}$ and $\mathbf{B}$ are the current density and magnetic field and $\mathbf{A}$ is the vector potential.

To obtain some physical insight of these structures, let us consider the special situation for the auroral region and/or the solar wind and make the reasonable assumption that the perturbed magnetic field fluctuations are much smaller than and essentially transverse to the mean magnetic field $B_{0}$ (which will be temporarily assumed to be uniform for the current discussion). Thus, let us write $\mathbf{B}=\left(\delta B_{x}, \delta B_{y}, B_{0}\right)$, where $\mathrm{z}$ is in the direction of the mean magnetic field, and $(\mathrm{x}, \mathrm{y})$ are orthogonal coordinates normal to $\mathrm{z}$. The force-free condition for constant $B_{0}$ and $\nabla \cdot \mathbf{J}=\mathbf{0}$ then leads approximately to the scalar condition $\mathbf{B} \cdot \nabla J_{z}=0$, obtained by taking the z-component of the curl of $\mathbf{J} \times \mathbf{B}=0$ (Rutherford, 1973; Tetreault, 1992b; Chang, 1998a,b,c). It can be shown that, with the inclusion of the kinetic effects through the anisotropic pressure terms and the generalized Ohm's law, the above results are still approximately valid. We have, then, approximately,

$$
B_{0} \partial J_{z} / \partial z=-\left(\delta B_{x} \partial / \partial x+\delta B_{y} \partial / \partial y\right) J_{z}+\cdots
$$

where the ellipsis represents the other modifying effects. For convenience, let us introduce the flux function $\psi$ by writing $(\partial \psi / \partial y,-\partial \psi / \partial x)$ $=\left(\delta B_{x}, \delta B_{y}\right)$ for the perturbed transverse components of the magnetic field in the $(x, y)$ directions such that $\nabla \cdot \mathbf{B}=0$ is satisfied. Then, $J_{z}$ and $\psi$ are governed by Eq. (1.2) and the Ampere's law (neglecting the modifying effects represented by the ellipsis).

A simple example of the flux function and axial current density satisfying the above conditions would be the class of circularly cylindrical solutions of $\psi(r)$ and $J_{z}(r)$. Generally, the solutions would be more involved because 
of the variabilities of the local conditions of the plasma and the threedimensional geometry. Moreover, the dynamic coherent structures with the inclusion of plasma pressure and other modifying effects (including electroninertia terms) would be even more complicated. However, we expect these structures to be usually in the form of field-aligned flux tubes, Fig. 1-1.

Generally, there exist various types of propagation modes (whistler modes, lower hybrid waves, etc.) in a magnetized plasma. Thus, we envision a corresponding number of different types of plasma resonances and associated coherent structures that typically characterize the dynamics of the plasma medium under the influence of a background magnetic field.

Generally, such coherent structures may take on the shapes of convective forms, nonlinear solitary structures, pseudo-equilibrium configurations, as well as other types of spatiotemporal varieties. Some of them may be more stable than the others. These spatiotemporal structures, however, generally are not purely laminar entities as they are composed of bundled fluctuations of all frequencies. Because of the nature of the physics of complexity, it will be futile to attempt to evaluate and/or study the details and stabilities of each of these infinite varieties of structures; although some basic understanding of each type of these structures will generally be helpful in the comprehension of the full complexity of the underlying nonlinear plasma dynamics.

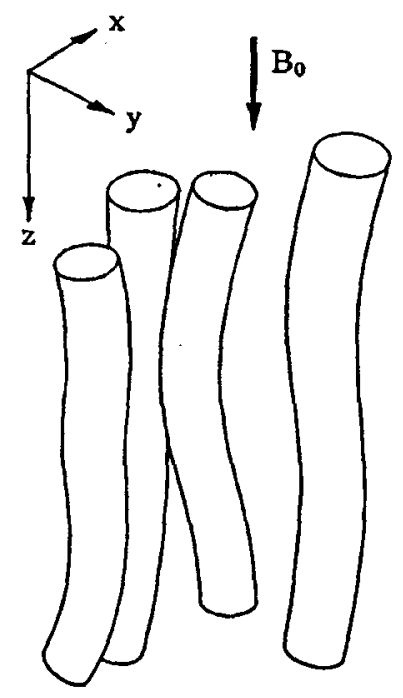

Figure -1. Field-aligned spatiotemporal coherent structures.

These coherent structures will wiggle, migrate, deform and undergo different types of motions and interactions under the influence of the local plasma and magnetic topologies. In the next section, we will consider how 
PLASMAS

the coherent structures can interact and produce the type of intermittency generally observed in a complex dynamical plasma.

\section{Complex Interactions of The Coherent Structures}

When coherent magnetic flux tubes of the same polarity migrate toward each other, strong local magnetic shears are created, Figs. 2-1 and 2-2. It has been demonstrated by $\mathrm{Wu}$ and Chang $(2000 \mathrm{a}, \mathrm{b} ; 2001)$ that existing sporadic non-propagating fluctuations will generally migrate toward the strong local shear region. Eventually the mean local energies of the coherent structures will be dissipated into these concentrated fluctuations in the coarse-grained sense.

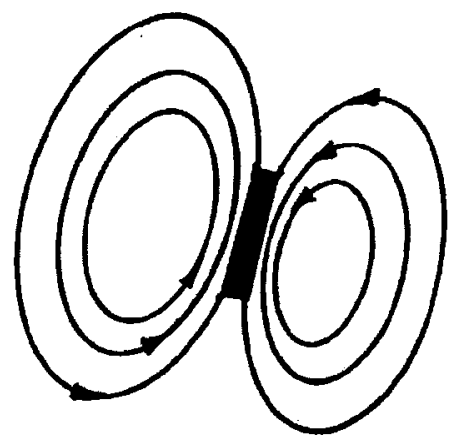

Figure 2-1. Cross-sectional view of coherent structures of the same polarity. Contours are $\psi=$ constants and arrows indicate directions of magnetic field in the $(x, y)$-plane. Blackened area is an intense current sheet.

Such enhanced intermittency at the intersection regions has been observed by Bruno et al. $(2001 ; 2002)$ in the solar wind using the tools of wavelet analyses and local intermittency measure (LIM). The coarsegrained dissipation will then initiate "fluctuation-induced nonlinear instabilities" (Chang, 1999; Chang et al., 2002); and, thereby reconfigure the topologies of the coherent structures into a combined lower local energetic state, eventually allowing the coherent structures to merge locally. On the other hand, when coherent structures of opposite polarities approach each other due to the forcing of the surrounding plasma, they might repel each other, scatter, or induce magnetically quiescent localized regions. Under any of the conditions of the above interaction scenarios, new fluctuations will be generated. And, these new fluctuations can provide new resonance sites; thereby nucleating new coherent structures of varied sizes. 

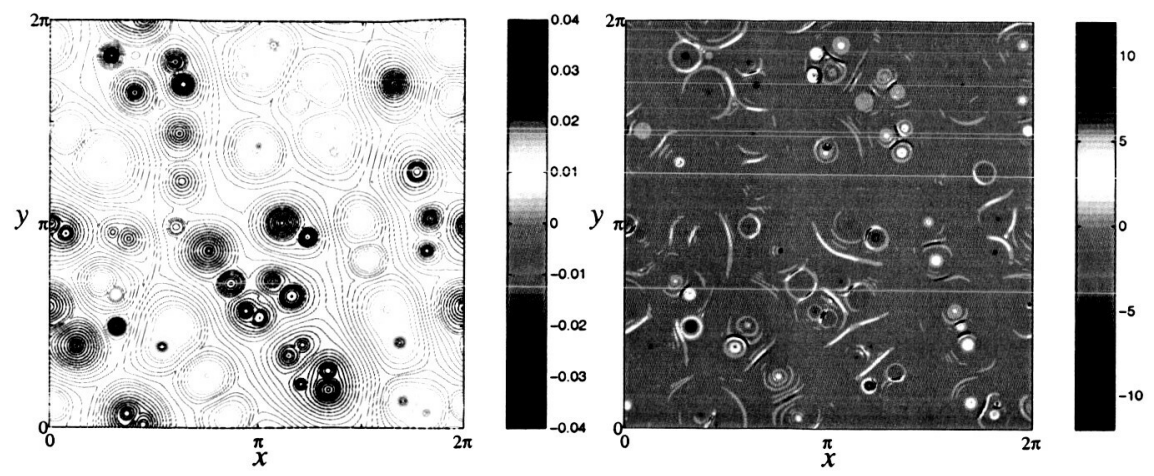

Figure 2-2. 2D MHD simulation of coherent structures (left panel) and current sheets (right panel) generated by initially randomly distributed current filaments after an elapsed time of $t=300$ units. (For reference, the sound wave and Alfvén wave traveling times through a distance of $2 \pi$ are approximately 4.4 and 60 , respectively.)

All such interactions can occur at any location of a flux tube along its field-aligned direction, and the phenomenon is fully three-dimensional. In order to gain some insight of the physical picture of the overall dynamics of the interactions of the coherent structures, let us again consider the auroral zone or the solar wind as an illustrative example. We make the plausible assumption that some aspects of the plasma dynamics may be approximately understood in terms of the formulation of reduced magnetohydrodynamics (RMHD) (Seyler, 1988; Matthaeus et al., 1990). In this approximation, we assume that the mean magnetic field is much larger than the transverse fields, and the field-aligned fluctuations of the magnetic and velocity components are much smaller than their transverse counterparts. As a consequence, the density of the plasma is uniform. Writing the equations in SI units with $\rho=1$ and $\mu_{0}=1$, we have (Strauss, 1976; Biskamp, 1993):

$$
d \psi / d t=B_{z} \partial \phi / \partial z, \quad d \omega / d t=\mathbf{B} \cdot \nabla j
$$

where $\psi(x, y)$ is the transverse flux function defined by $\mathbf{B}=\mathbf{e}_{z} \times \nabla \psi+B_{z} \mathbf{e}_{z}, \phi(x, y)$ is the transverse stream function defined by $\mathbf{v}_{\perp}=\mathbf{e}_{z} \times \nabla \phi$, and $\omega=\nabla_{\perp}^{2} \phi$ is the vorticity, $j=\nabla_{\perp}^{2} \psi$ is the field-aligned current density with $d / d t=\partial / \partial t+\mathrm{v} \cdot \nabla$. The equations are written in the moving frame along the mean magnetic field direction $z$, and $(x, y)$ are the transverse orthogonal directions.

From Equations (2.1), we note that the primary nonlinear interactions occur generally in the transverse direction to the mean magnetic field. And, the coupling in the field-aligned direction is essentially linear. Thus, fluctuations generated by the transverse nonlinear interactions will scatter 
and evolve nonlinearly primarily in the transverse direction. At this point, we realize that the RMHD formulation is too restrictive as some of the interactions of the flux tubes may become more oblique and thereby allowing the fluctuations to attain a broader range of values of $k_{\|}$than otherwise would have been admitted by the RMHD approximation. Thus, a significant amount of the fluctuations generated by the interactions can become commensurate with the plasma dispersion relation and propagate in the field-aligned direction as Alfvén waves due to this three-dimensional complexity-induced enhanced transport. Eventually a dynamic topology of a complex state of coexisting propagating and non-propagating magnetic fluctuations is created. In the auroral region, the plasma may be electroninertia dominated and the above discussion can be easily generalized to include such kinetic effects.

\section{Invariant Scaling and Topological Phase Transitions}

In the above sections, we provided some convincing arguments as well as numerical and observational evidences indicating that space plasma turbulence is generally in a state of topological complexity. By "complex" topological states we mean magnetic topologies that are not immediately . deducible from the elemental (e.g., MHD and/or Vlasov) equations (Consolini and Chang, 2001). Below, we shall briefly address the salient features of the analogy between topological and equilibrium phase transitions. A thorough discussion of these ideas may be found in Chang $(1992,1999 ; 2001$, and references contained therein).

For nonlinear stochastic systems exhibiting complexity, the correlations among the fluctuations of the random dynamical fields are generally extremely long-ranged and there exist many correlation scales. The dynamics of such systems are notoriously difficult to handle either analytically or numerically. On the other hand, since the correlations are extremely long-ranged, it is reasonable to expect that the system will exhibit some sort of invariance under coarse-graining scale transformations. A powerful technique that utilizes this invariance property is the method of the dynamic renormalization group (Chang et al., 1978; 1992; and references contained therein). - The technique is a generalization of the static renormalization group introduced by Wilson (Wilson and Kogut, 1974).

As it has been demonstrated by Chang et al. (1978), based on the path integral formalism, the behavior of a nonlinear stochastic system far from equilibrium may be described in terms of a "stochastic Lagrangian $L$ ", such that the probability density functional $P$ of the stochastic system is expressible as: 


$$
P(\varphi(\mathbf{x}, t))=\int D[\chi] \exp \left\{-i \int L(\dot{\varphi}, \boldsymbol{\varphi}, \chi) d \mathbf{x} d t\right\}
$$

where $\varphi(\mathbf{x}, t)=\phi_{i}(i=1,2, \ldots, N)$ are the stochastic variables such as the fluctuating magnetic, velocity and electric fields, and $\chi(x, t)=$ $\chi_{i}(i=1,2, \ldots, N)$ are the conjugate stochastic momentum variables that may be rigorously derived from the underlying stochastic equations governing $\varphi$ (Chang et al., 1978; Chang, 1992; Chang et al., 1992).

Then, the renormalization-group (coarse-graining) transformation may be formally expressed as:

$$
\partial L / \partial \ell=R L
$$

where $R$ is the renormalization-group (coarse-graining) transformation operator and $\ell$ is the coarse-graining parameter for the continuous group of transformations. It will be convenient to consider the state of the stochastic Lagrangian in terms of its parameters $\left\{P_{n}\right\}$. Equation (3.2), then, specifies how the Lagrangian, $L$, flows (changes) with $\ell$ in the affine space spanned by $\left\{P_{n}\right\}$, Fig. 3-1.

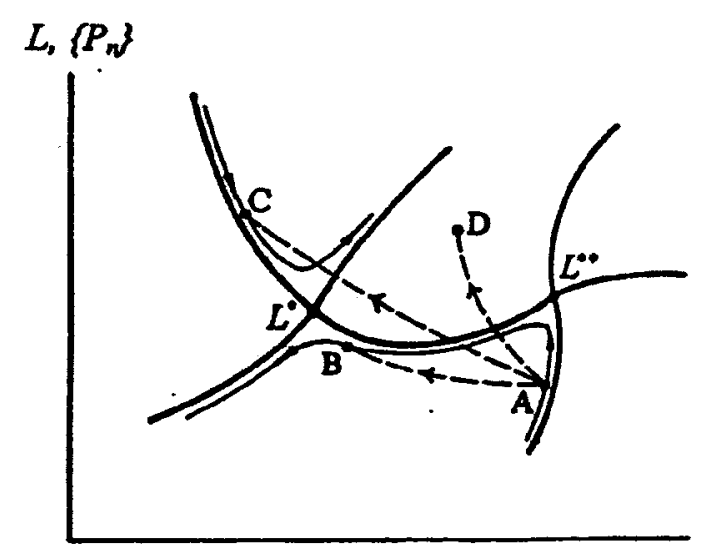

Figure 3-1. Renormalization-group trajectories and fixed points.

Forced and/or Self-Organized Criticality. Generally, there exists a number of fixed points (singular points) in the flow field, at which $d L / d \ell=0$. At each such fixed point ( $L^{*}$ or $L^{* *}$ in Fig. 3-1), the correlation length should not be changing. However, the renormalization-group transformation requires that all length scales must change under the coarsegraining procedure. Therefore, to satisfy both requirements, the correlation 
PLASMAS

length must be either infinite or zero. When it is at infinity, the dynamical system is then at a state of forced and/or self-organized criticality (FSOC) (Bak et al., 1987; Chang, 1992), analogous to the state of criticality in equilibrium phase transitions (Stanley, 1971). To study the stochastic behavior of a nonlinear dynamical system near such a dynamical critical state (e.g., the one characterized by the fixed point $L^{*}$ ), we linearize the renormalization-group operator $R$ about $L^{*}$. The mathematical consequence of this approximation is that, close to dynamic criticality, certain linear combinations of the parameters that characterize the stochastic Lagrangian $L$ will correlate with each other in the form of power laws. These include, in particular, the $(k, \omega)$, i.e. mode number and frequency, spectra of the correlations of the various fluctuations of the dynamic field variables.

Such power law behavior has been detected in the probability distributions of solar flare intensities ( $\mathrm{Lu}, 1995)$, in the AE burst occurrences as a function of the AE burst strength (Consolini, 1997), in the global auroral UVI imagery of the statistics of size and energy dissipated by the magnetospheric system (Lui et al., 2000), in the probability distributions of spatiotemporal magnetospheric disturbances as seen in the UVI images of the nighttime ionosphere (Uritsky et al., 2002), and in the probability distributions of durations of Bursty Bulk Flows (Angelopoulos, 1999); although some of the above interpretations of observed data may, however, also be amenable to alternative explanations (Boffetta et al., 1999; Freemanet al., 2000; Watkins, 2002).

In addition, it can be demonstrated from such a linearized analysis of the dynamic renormalization group that generally only a small number of (relevant) parameters are needed to characterize the stochastic state of the system near criticality (Chang, 1992) justifying the recent work suggesting that certain dynamic characteristics of the magnetotail could be modeled by the deterministic chaos of low-dimensional nonlinear systems (Baker et al., 1990; Klimas et al., 1992; Sharma et al., 1993).

Illustrative Examples. The intermittency description for plasma turbulence of fluctuations may be modeled by the combination of a localized chaotic functional growth equation of a set of relevant order parameters and a functional transport equation of the control parameters (Chang and $\mathrm{Wu}$, 2002; Chang et al., 2003). Below, we shall provide two simple phenomenological models, which may have some relevance to the auroral zone or the solar wind (Chang, 2003).

Model I. Assuming that the parallel mean magnetic field $B_{0}$ is sufficiently strong and the magnetic fluctuations dominate in the transverse directions, we introduce the flux function $\psi$ for the transverse fluctuations as follows, 


$$
\mathbf{B}=\mathbf{e}_{z} \times \nabla \psi+B_{0} \mathbf{e}_{z}
$$

The coherent structures for such a system are generally flux tubes approximately aligned in the mean parallel direction (Chang, 2001). Conservation of helicity (e.g., under the RMHD approximation) indicates that the integral of $\psi$ over a flux tube is approximately constant. Instead of invoking the RMHD formalism, however, here we simply consider $\psi$ as an order parameter. As the flux tubes merge and interact, they may correlate over long distances, which, in turn, will induce long relaxation times near FSOC (Chang, 1992). Let us assume that the transverse size of the system is sufficiently broad compared to the cross sections of the coherent structures (or flux tubes), such that we may invoke homogeneity and assume the dynamics to be independent of boundary effects. We may then model the dynamics of flux tube mergings and interactions, in the crudest approximation, in terms of the following order-disorder intermittency equation:

$$
\partial \psi_{k} / \partial t=-\Gamma_{k} \partial F / \partial \psi_{-k}+f_{k}
$$

where $\psi_{k}$ are the Fourier components of the flux function, $\Gamma_{k}$ an analytic function of $k^{2}, F\left(\psi_{k}, k\right)$ the state function, and $f_{k}$ a random noise which includes all the other effects that are not included in the first two terms of this crude model.

Model $\boldsymbol{I I}$. In the above model, we have neglected both the effects of diffusion and convection. We next construct a phenomenological model that includes the transport of cross-field diffusion. We now assume the state function to depend on the flux function $\psi$ and the local pseudo-energy measure $\xi$. Thus, in addition to the dynamic equation (3.4), we now also include a diffusion equation for $\xi$. In Fourier space, we have

$$
\partial \xi_{k} / \partial t=-D k^{2} \partial F / \partial \xi_{-k}+h_{k}
$$

where $\xi_{k}$ are the Fourier components of $\xi, D(k)$ is the diffusion coefficient, and the state function is now $F\left(\psi_{k}, \xi_{k}, k\right)$, and $h_{k}$ is a random noise. By doing so, we separate the slow pseudo-energy transport due to diffusion of the local pseudo-energy measure $\xi$ from the noise term of (3.4). We note that an approach similar to these ideas have been considered by Klimas et al. (2000).

Dynamic Renormalization-Group Analysis. We have performed renormalization-group analyses as outlined above for the two kinetic models 
PLASMAS

described above. We note that under the dynamic renormalization-group (DRG) transformation, the correlation function $C$ of $\psi_{k}$ should scale as:

$$
e^{a_{c} \ell} C(k, \omega)=C\left(k e^{\ell}, \omega e^{a_{\omega} \ell}\right)
$$

where $\omega$ is the Fourier transform of $t, \ell$ the renormalization parameter as defined in the previous section, and $\left(a_{c}, a_{\omega}\right)$ the correlation and dynamic exponents. Thus, $C / \omega^{a_{c}} / a_{\omega}$ is an absolute invariant under the DRG, or $C \sim \omega^{-\lambda}$, where $\lambda=-a_{c} / a_{\omega}$. DRG analysis of Model I with Gaussian noise yields the value of $\lambda$ to be approximately equal to 2.0. DRG analyses performed for Model II for Gaussian noises for several approximations yield the value for $\lambda$ to be approximately equal to 1.88 to 1.66 .

Interestingly, for both models, DRG calculations give an approximate value of -1.0 for the $\omega$-exponent for the trace of the transverse magnetic correlation tensor. Matthaeus and Goldstein (1986) had suggested that such an exponent might represent the superposition of discrete structures emerged from the solar convection zone; thereby giving some credence to the above modeling effort. Also, the corresponding $k$-exponent is found to be approximately equal to -2 for both models. These results compare rather favorably with the results of our 2D MHD numerical simulations, Fig. 3-2.

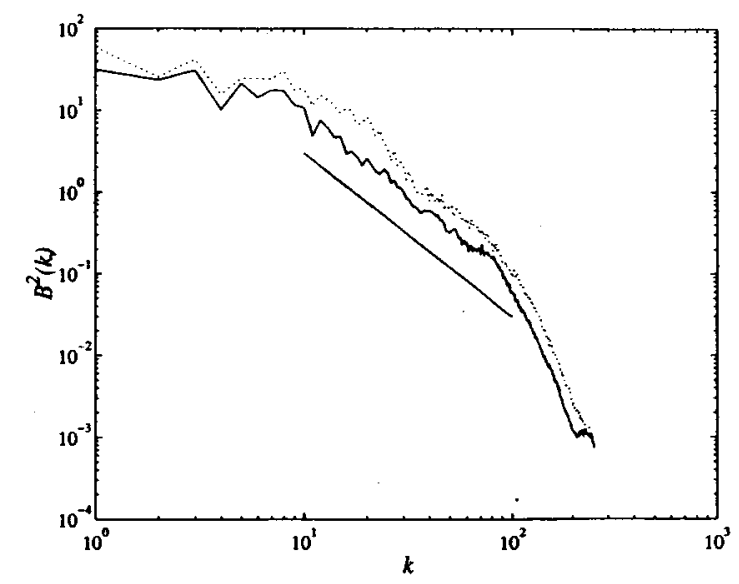

Figure 3-2. Fourier spectrum of $B^{2}(k)$ at $t=300$ (dotted) and 600 (solid). Solid straight line indicates a slope of $\mathbf{- 2}$.

Symmetry breaking and Topological Phase Transitions. As the dynamical system evolves in time (autonomously or under external forcing), the state of the system (i.e., the values of the set of the parameters 
characterizing the stochastic Lagrangian, $L$ ) changes accordingly. A number of dynamical scenarios are possible. For example, the system may evolve from a critical state A (characterized by $L^{* *}$ ) to another critical state $\mathrm{B}$ (characterized by $L^{*}$ ) as shown in Fig. 3-1. In this case, the system may evolve continuously from one critical state to another. On the other hand, the evolution from the critical state $A$ to critical state $C$ as shown in Fig. 3-1 would probably involve a dynamical instability characterized by a firstorder-like topological phase transition (fluctuation-induced nonlinear instability) because the dynamical path of evolution of the stochastic system would have to cross over a couple of topological (renormalization-group) separatrices. For such a situation the underlying magnetic topology and its related plasma state will generally undergo drastic changes. Similar ideas along these lines have been advanced by Sitnov et al. (2000) and simulated based on the cellular automata calculations of sandpile models (Chapman et al., 1998; Watkins et al., 1999).

Under either of these above scenarios, the spectra indices will generally change either continuously or abruptly. Such type of multifractal phenomena is commonly observed in the magnetotail, the auroral zone, and the solar wind (Lui, 1998; Hoshino et al., 1994; Milovanov et al., 1996; André and Chang, 1992; Chang, 2001; Bruno et al., 2001; 2002; Tu and Marsch, 1995; and references contained therein.)

Alternatively, a dynamical system may evolve from a critical state $A$ to a state D (as shown in Fig. 3-1) which may not be situated in a regime dominated by any of the fixed points; in such a case, the final state of the system will no longer exhibit any of the characteristic properties that are associated with dynamic criticality. As another possibility, the dynamical system may deviate only moderately from the domain of a critical state characterized by a particular fixed point such that the system may still display low-dimensional scaling laws, but the scaling laws may now be deduced from straightforward dimensional arguments. The system is then in a so-called mean-field state. (For general references of symmetry breaking and nonlinear crossover, see Chang and Stanley (1973); Chang et al. (1973a; 1973b); Nicoll et al. $(1974 ; 1976)$.)

Experimental observations of plasma fluctuations in the Sun-Earth connection region generally yield broken power law spectra similar to those displayed in Fig. 3-2 of the 2D numerical simulation results. Such abrupt changes of scaling powers of the k-spectra are signatures of symmetry breaking. The broken symmetries may be due to the abrupt change of the degree of intermittency of fluctuations from large to small scales, or due to the change of the underlying physics (e.g., from MHD to kinetic processes), or variations of external forcing, or finite boundaries and other effects. 


\section{Intermittency}

Nearly all fluctuations in space plasmas exhibit intermittency. For turbulent dynamical systems with intermittency, the transfer of energy (or other relevant scalars and tensors) due to fluctuations from one scale to another deviates significantly from uniformity. A technique of measuring the degree of intermittency is the study of the departure from Gaussianity the probability distribution functions of turbulent fluctuations at different scales. To demonstrate this point, let us refer to the 2D numerical simulation results described in Section 2. For example, we may generate the probability distribution function $P\left(\delta B^{2}, \delta\right)$ of $\delta B^{2}(x, \delta) \equiv B^{2}(x+\delta)-B^{2}(x)$ at a given time $\mathrm{t}$ for such simulations, where $\delta$ is the scale of separation in the $\mathrm{x}$-direction. Figure 4-1 displays the calculated results of $P\left(\delta B^{2}, \delta\right)$ from a numerical simulation for several scales $\delta$.

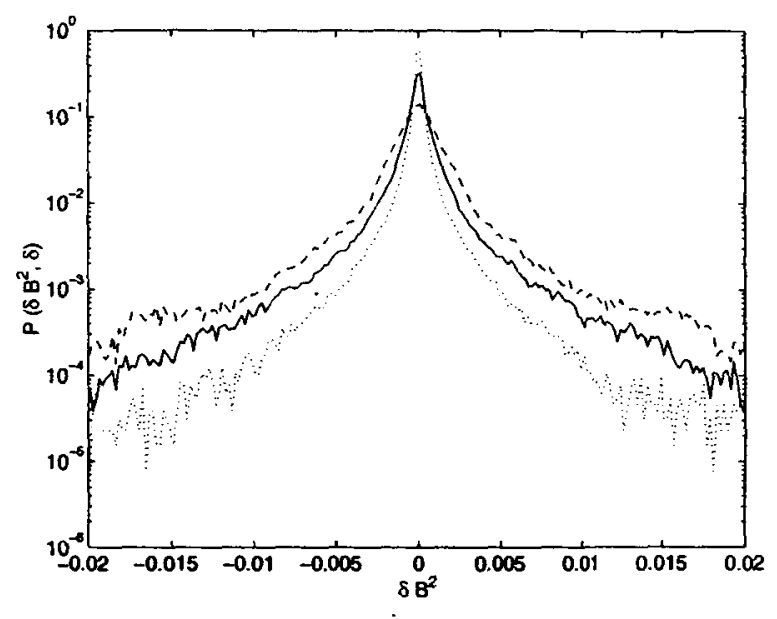

Figure 4-1. 2D MHD simulation result at $t=300$ of PDF's of $B^{2}$ at scales of 2 (dotted), 8 (solid), and 32 (dashed) units of grid spacing $\mathcal{E}$.

From this figure, we note that the deviation from Gaussianity becomes more and more pronounced at smaller and smaller scales. In an interesting paper by Hnat et al. (2002), they demonstrated that such probability distributions for solar wind fluctuations exhibit approximate mono-power scaling according to the following functional relation:

$$
P\left(\delta B^{2}, \delta\right)=\delta^{-s} P_{s}\left(\delta B^{2} \delta^{-s}, \delta\right)
$$


parameters in general and may sometimes, for example, be represented by the $\kappa$ - or Castaing distributions (Sorriso-Valvo et al., 1999; Jurac, 2003; Forman and Burlaga, 2003; Weygand, 2003; Castaing et al., 1990). Their scaling properties are more subtle and will not be considered in this brief review.

Since the degree of intermittency generally increases inversely with scale, it will be interesting to study the degrees of intermittency locally at different scales. This can be accomplished by the method of Local Intermittency Measure (LIM) using the wavelet transforms. A wavelet transform generally is composed of modes which are square integrable localized functions that are capable of unfolding fluctuating fields into space and scale (Farge, 1992). Figure 4-3b is the power spectrum of a complex Morlet wavelet transform of the current density for one of the 2D MHD simulations mentioned in Section 2. We notice that the intensity of the current density is sporadic and varies nonuniformly with scale.

We now define $\operatorname{LIM}(1)$ as the ratio of the squared wavelet amplitude $|\psi(x, \delta)|^{2}$ and its space averaged value $\left\langle|\psi(x, \delta)|^{2}>_{x}\right.$. We note that $\operatorname{LIM}(1)=1$ for the Fourier spectrum. To emphasize the variation of intensity with scale, we also consider the logarithm of LIM(1). It has been suggested by Meneveau (1991) that the space average of the square of LIM(1), which is a scale dependent measure of the kurtosis or flatness, is a convenient gauge of the deviation of intermittency from Gaussianity. We denote this measure by $\operatorname{LIM}(2)$. It is equal to 3 if the probability distribution is Gaussian. Figures 4-3d,e and 4-4 are graphical displays of the calculated results of $\operatorname{LIM}(1), \operatorname{logLIM}(1)$ and $\operatorname{LIM}(2)$ for our $2 \mathrm{D}$ numerical simulations using the complex Morlet transform. We notice that the fluctuations are indeed scale dependent, localized and strongly intermittent at small scales. Similar experimental results using the wavelet transforms have been found, for example, by Consolini et al. (2004) for the magnetotail and Bruno et al. (2001) for the solar wind.

In the above, we considered some simplified models and numerical examples that may have some relevance to intermittent turbulence in space plasmas. Realistic models for these phenomena will generally be much more complicated. For example, from the RMHD formulation of (2.1), we recognize that there should at least be two competing order parameters. These are the flux function $\psi$ and the stream function $\phi$ (which is linearly proportional to the electrostatic potential). Thus, the intermittency equation (such as (3.4)) needs to be generalized to accommodate these coupled order parameters. Within the RMHD formulation, there exist useful Hamiltonian and operator-algebra structures (Morrison and Hazeltine, 1984), which should prove invaluable in developing the generalized state function $F$ of the 
where $s$ is the mono-scaling power. We demonstrate that mono-power scaling also holds approximately for our simulated results with the value of $s$ equal to approximately 0.335 .

The reason for mono-power scaling for $\delta B^{2}$ may be understood in terms of the renormalization-group arguments presented in Section 3. If we assume that $\delta B^{2}$ is one of the relevant eigenoperators near a critical fixed point, then the probability distribution function for $P\left(\delta B^{2}, \delta\right), \delta B^{2}$, as well as $\delta$ will scale linearly as follows:

$$
P^{\prime}=P \exp \left(a_{p} l\right), \quad \delta B^{2^{\prime}}=\delta B^{2} \exp \left(a_{B^{2}} l\right), \quad \delta^{\prime}=\delta \exp \left(a_{\delta} l\right)
$$

where $\left(a_{p}, a_{B^{2}}, a_{\delta}\right)$ are scaling powers. Thus, we obtain two irreducible absolute invariants: $P / \delta^{a_{P} / a_{\delta}}$ and $\delta B^{2} / \delta^{a_{B^{2}} / a_{\delta}}$. Since $P=P\left(\delta B^{2}, \delta\right)$, there must be a functional relation between these two invariants (Chang et al., 1973a,b). Therefore, we obtain the following scaling relation among $\left(P, \delta B^{2}, \delta\right): P / \delta^{a_{P} / a_{\delta}}=F\left(\delta B^{2} / \delta^{a_{B^{2}} / a_{\delta}}\right)$.

Without loss of generality, we may choose $a_{\delta}=1$. With the additional constraint that the probability distribution functions are normalized, we immediately obtain the expression of Hnat et al. as shown in (4.1), Fig. 4-2.

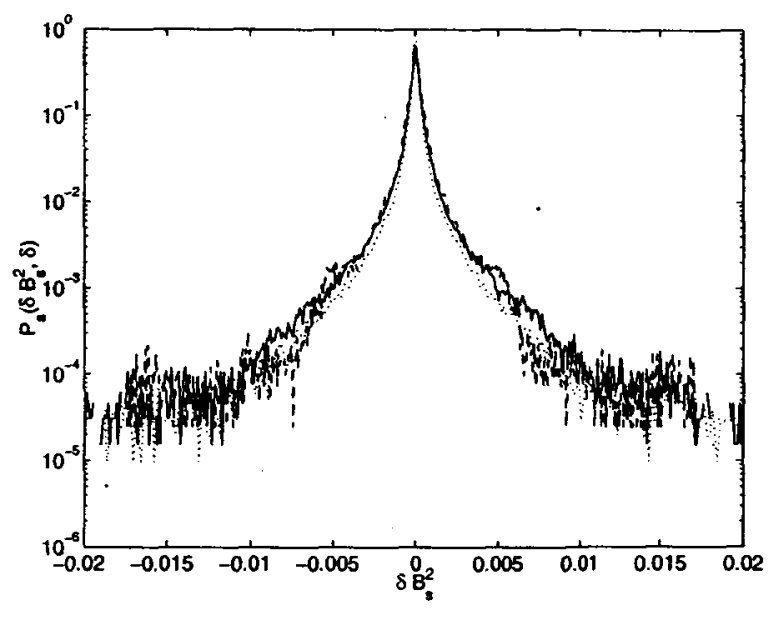

Figure 4-2. Scaled PDF's according to Eq. (4.1) with $s=0.335$. Line styles are the same as in Figure 4-1.

Actually the scaling relation (4.1) is approximate in that the tails of the distributions in Fig. 4-2 do not exactly fall onto one curve. This is the intrinsic nature of the strong intermittency at small scales. Thus, representations of the probability density functions will involve multi- 

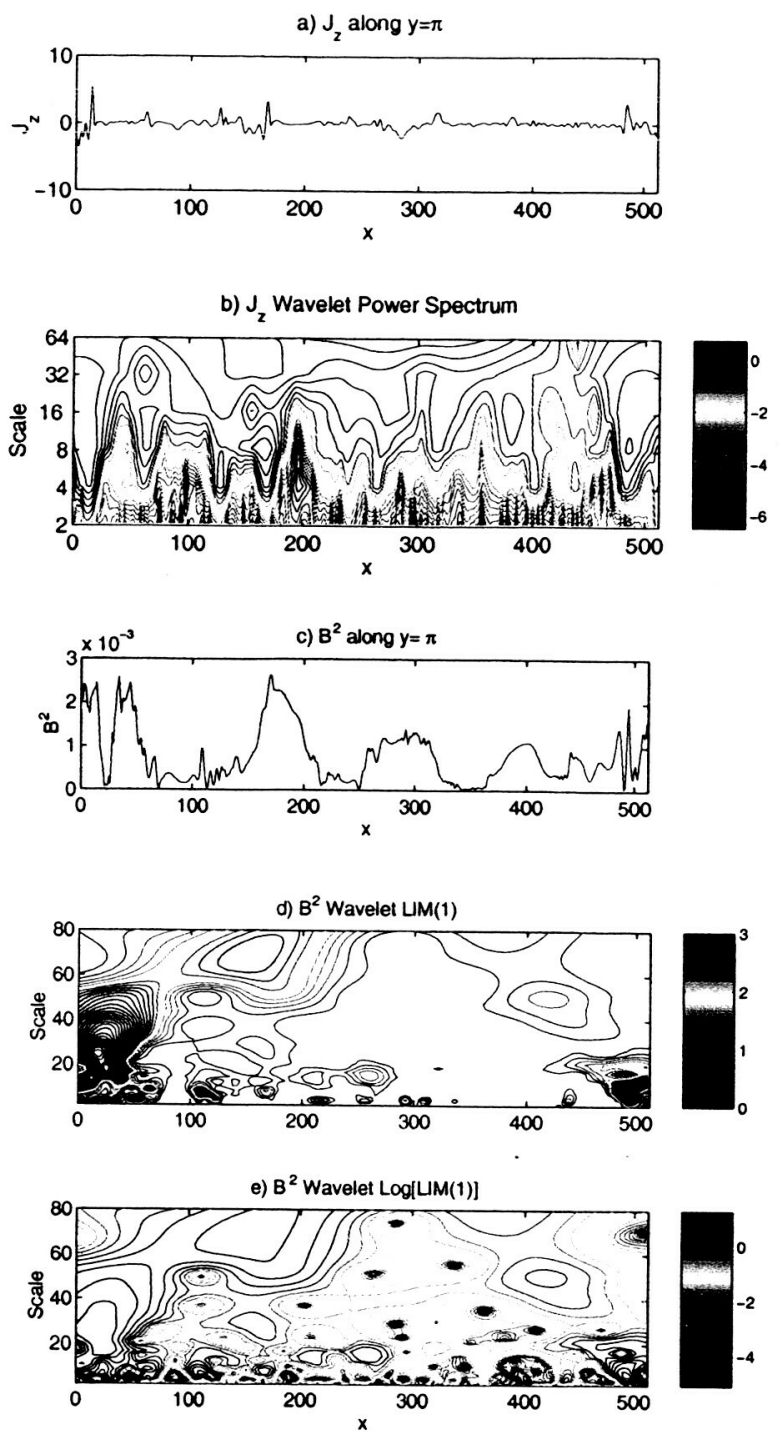

Figure 4-3. (a) 2D MHD simulation result of current density $J_{z}$ along $y$-axis at $t=300$. $\begin{array}{lll}\text { (b) Power spectrum of complex Morlet wavelet transform of } J_{z} & \text { (c) 2D MHD simulation }\end{array}$ result of $B^{2}$ distribution along the $x$-axis for $y=\pi$ at $t=300$. (d and e) Contour plots of $\operatorname{LIM}(1)$ and $\operatorname{LogLIM}(1)$ of $B^{2}$. The $x$-axis and scale are in units of the grid spacing $\varepsilon$. 


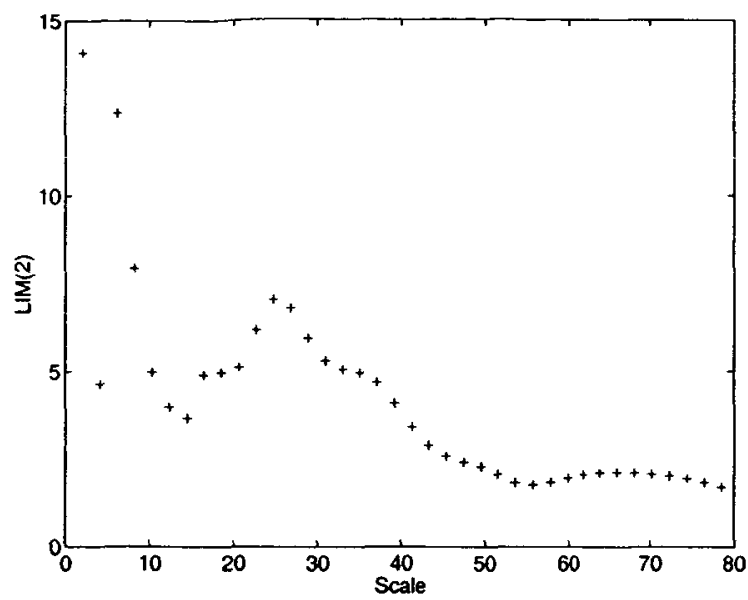

Figure 4-4. LIM(2) of $B^{2}$ for the same $B^{2}$ distribution of Figure 4-3c.

coupled order parameters and the state variables as well as the intermittency equation itself. Formulations of coupled order parameters and their related theoretical analyses for a variety of criticality problems in condensed matter physics have been considered by Chang et al. (1992; and references contained therein).

In addition, the transport equation such as (3.5) for the global system should generally also include convection and acceleration terms in addition to that of diffusion (Chang et al., 2003; 2004). Thus, at the minimum our model transport equation must take on the form of the RMHD (3.5) with the addition of "coarse-grained" dissipation terms, which generally will be functionals of the coupled order parameters. It should also contain terms representing the complexity-induced enhanced field-aligned transport. These generalizations will not be considered in this brief review.

\section{Energization of Ions by Intermittent Fluctuations in The Auroral Zone}

It has long been recognized that the commonly observed broadband, low frequency electric field fluctuations are responsible for the acceleration of oxygen ions in the auroral zone. In order for the fluctuating electric field to resonantly accelerate the ions continuously as the ions evolve upward along the field lines, they must be in continuous resonance with the ions. There did not seem to exist a fully viable mechanism that can generate a spectrum of fluctuations broadband and incoherent enough to fulfill this stringent requirement. 
Assuming that the RMHD formulation holds approximately in the auroral zone, the electrostatic fluctuations transverse to the field-aligned direction are given approximately by the velocity fluctuations: $\mathbf{v} \times B_{z} \mathbf{e}_{z}$. The ordering due to the stream function $\phi$ may be important in the auroral zone and therefore, the electrostatic fluctuations can be quite significant there. Because of the small scales involved, the dynamic intermittency produced by the merging and interactions of the coherent structures are probably generated by the whistler turbulence, electron-inertia related tearing modes, and/or other collisionless modes (Chang, 2001). Therefore, a significant portion of the fluctuations would be kinetic. Nevertheless, the electric field fluctuations would still be predominantly transverse and electrostatic. Thus, the low frequency fluctuations commonly observed in the auroral zone are probably contributed by these non-propagating intermittent fluctuations intermingled with a small fraction of propagating modes.

Below, we shall briefly discuss how such fluctuations can efficiently energize the oxygen ions from ionospheric to magnetospheric energies. Assuming the oxygen ions are test particles, they would respond to the transverse electric field fluctuations $\mathbf{E}_{\perp}$ near the oxygen gyrofrequency locally according to the Langevin equation:

$$
d \mathbf{v}_{\perp} / d t=q_{i} \mathbf{E}_{\perp} / m_{i}
$$

To understand the stochastic nature of the Langevin equation, we visualize an ensemble of ions $f\left(\mathbf{v}_{\perp}\right)$ and study its stochastic properties. Assuming that the interaction times among the particles and the local electric field fluctuations are small compared to the global evolution time, we may write within the interaction time scale:

$$
f\left(\mathbf{v}_{\perp}, t+\Delta t\right)=\int f\left(\mathbf{v}_{\perp}-\Delta \mathbf{v}_{\perp}, t\right) P_{t}\left(\mathbf{v}_{\perp}-\Delta \mathbf{v}_{\perp}, \Delta \mathbf{v}_{\perp}\right) d \Delta \mathbf{v}_{\perp}
$$

where $P_{t}\left(v_{\perp}-\Delta v_{\perp}, \Delta v_{\perp}\right)$ is the normalized transition probability of a particle whose velocity changes from $v_{\perp}-\Delta v_{\perp}$ to $v_{\perp}$ in $\Delta t$, and $\Delta v_{\perp}$ ranges over all possible magnitudes and transverse directions. Standard procedure at this point is to expand both sides of (5.2) in Taylor series expansions:

$$
\begin{aligned}
\frac{\partial f}{\partial t} \Delta t+O\left((\Delta t)^{2}\right)= & -\frac{\partial}{\partial \mathbf{v}_{\perp}} \cdot\left[\left\langle\Delta \mathbf{v}_{\perp}\right\rangle f\right] \\
& +\frac{1}{2} \frac{\partial^{2}}{\partial \mathbf{v}_{\perp} \partial \mathbf{v}_{\perp}}:\left[\left\langle\Delta \mathbf{v}_{\perp} \Delta \mathbf{v}_{\perp}\right\rangle f\right]+O\left(\left(\Delta \mathbf{v}_{\perp}\right)^{3}\right)
\end{aligned}
$$


where

$$
\begin{aligned}
& \left\langle\Delta \mathbf{v}_{\perp}\right\rangle=\int P_{l}\left(\mathbf{v}_{\perp}, \Delta \mathbf{v}_{\perp}\right) \Delta \mathbf{v}_{\perp} d\left(\Delta \mathbf{v}_{\perp}\right) \text {, and } \\
& \left\langle\Delta \mathbf{v}_{\perp} \Delta \mathbf{v}_{\perp}\right\rangle=\int P_{l}\left(\mathbf{v}_{\perp}, \Delta \mathbf{v}_{\perp}\right) \Delta \mathbf{v}_{\perp} \Delta \mathbf{v}_{\perp} d\left(\Delta \mathbf{v}_{\perp}\right)
\end{aligned}
$$

If we assume the $O\left(\left(\Delta \mathbf{v}_{1}\right)^{3}\right)$ terms are of order $(\Delta t)^{2}$ or higher, then in the limit of $\Delta t \rightarrow 0$, we obtain a Fokker-Planck equation (Einstein, 1905; Chandrasekhar 1943), where the drift and diffusion coefficients are defined as: $\mathbf{D}_{1}=<\Delta \mathbf{v}_{1}>/ \Delta t$ and $\mathbf{D}_{2}=\left\langle\Delta \mathbf{v}_{1} \Delta \mathbf{v}_{1}>/ 2 \Delta t\right.$ in the limit of $\Delta t \rightarrow 0$. These coefficients may be calculated straightforwardly using the Langevin equation. If the transition probability $P_{l}$ is symmetric in $\Delta \mathbf{v}_{\perp}$, then $\mathbf{D}_{1}$ vanishes and (5.3) reduces to a diffusion equation in the transverse direction. We note that if the electric field fluctuations are Gaussian, then the higher order correlations of the fluctuations are automatically equal to zero.

We shall come back to the discussion of the effects of general intermittent fluctuations on particle energization processes. For the moment, let us assume the approach using the Fokker-Planck formulation is valid and proceed. Since we have assumed the time scale for the particle-fluctuation interactions is much smaller than the global evolution time of the ion populations, we may then write the steady-state global evolution equation along an auroral field line $s$ under the guiding center approximation and neglecting the cross-field drift as (Chang et al., 1986; Retterer et al., 1987; Crew and Chang, 1988):

$$
\begin{aligned}
\frac{\partial}{\partial s}\left[v_{\|} \frac{f}{B_{z}}\right]+\frac{\partial}{\partial v_{\|}}\left[-\frac{v_{\perp}^{2}}{2 B_{z}} \frac{d B_{z}}{d s} \frac{f}{B_{z}}\right]+\frac{1}{v_{\perp}} & \frac{\partial}{\partial v_{\perp}}\left[v_{\perp} \frac{v_{\perp} v_{\|}}{2 B_{z}} \frac{d B_{z}}{d s} \frac{f}{B_{z}}\right] \\
& =\frac{1}{v_{\perp}} \frac{\partial}{\partial v_{\perp}}\left[v_{\perp} D_{\perp} \frac{\partial}{\partial v_{\perp}} \frac{f}{B_{z}}\right]
\end{aligned}
$$

where $v_{\|}, v_{\perp}$ are the parallel and perpendicular components of the particle velocity with respect to the field-aligned direction. This expression may be interpreted as a convective-diffusion equation for the density of the guiding center ions per unit length of flux tube $f / B_{z}$, in the coordinate space of $\left(s, v_{\|}, v_{\perp}\right)$.

To evaluate $D_{1}$, the gyrotropic perpendicular diffusion coefficient, from $\mathbf{D}_{2}$, we recognize that the fluctuations are broadband both in $k_{\perp}$ and $\omega$. Therefore, at all times, some portion of the fluctuations will be in resonance with the ions. The resonance condition, however, is strongly dependent on 
the localization and scale dependency of the intermittent fluctuations (Chang, 2001). We demonstrate below, as a simple illustrative example, how such resonant interactions may be accomplished by neglecting the Doppler shifts due to $\mathbf{k}$ such that only the intermittent fluctuations clustering around the instantaneous gyrofrequency of the ions provide the main contributions to the diffusion process. Standard arguments then lead to the following expression for the perpendicular diffusion coefficient:

$$
D_{\perp}=\left(\pi q_{i}^{2} / 2 m_{i}^{2}\right)\left\langle\left|E^{2}\right|\left(\Omega_{i}\right)\right\rangle_{r}
$$

where $<\left|E^{2}\right|\left(\Omega_{i}\right)>_{r}$ is the resonant portion of the average of the square of the transverse electric field fluctuations evaluated at the instantaneous gyrofrequency of the ions, $\Omega_{i}$.

Measurements by polar orbiting satellites indicate that the electric field spectral density $\Sigma$ follows an approximate power law $\Sigma^{-\alpha}$ in the range of the local oxygen gyrofrequencies, where $\alpha$ is a constant. This is a natural consequence of the intermittent turbulence when the fluctuations are close to a state of forced and/or self-organized criticality. If we make the additional approximations by assuming that the spectrum observed at the satellite is applicable to all altitudes and choosing the geomagnetic field to scale with the altitude as $s^{-3}$, we would then expect $\Sigma\left(\Omega_{i}, s\right)$ to vary with altitude $s$ as $s^{3 \alpha}$. Because we have made some rather restrictive resonance requirements for the fluctuations to interact with the ions, we expect the resonant portion of the average of the square of the transverse electric field fluctuations to be only a fraction $\eta$ of the total measured electric field spectral density. Therefore, we arrive at the following approximate expression for the diffusion coefficient:

$$
D_{\perp}=\left(\eta \pi q_{i}^{2} / 2 m_{i}^{2}\right) \Sigma_{0}\left(s / s_{o}\right)^{3 \alpha}
$$

We have performed global Monte Carlo simulations for Equations (5.4) and (5.6) for the conic event discussed by Retterer et al. (1987) with $\alpha=1.7$ and $\Sigma_{0}=1.9 \times 10^{-7}(\mathrm{~V} / \mathrm{m})^{2} \mathrm{sec} / \mathrm{rad}$. Figure 5-1 shows the measured oxygen velocity distribution contours (top panel) along with the corresponding calculated contours for $\eta=1 / 8$ (bottom panel) at the satellite altitude of $s_{o}=2 R_{E}$. Thus, with one eighth of the measured electric field spectral density contributing, the broadband fluctuations can adequately generate an oxygen distribution function with the energy and shape comparable to that obtained from observations. We have also calculated the oxygen ion distributions for a range of altitudes under the same conditions. Figure 5-2 is a plot of the average parallel energy versus the average perpendicular energy 

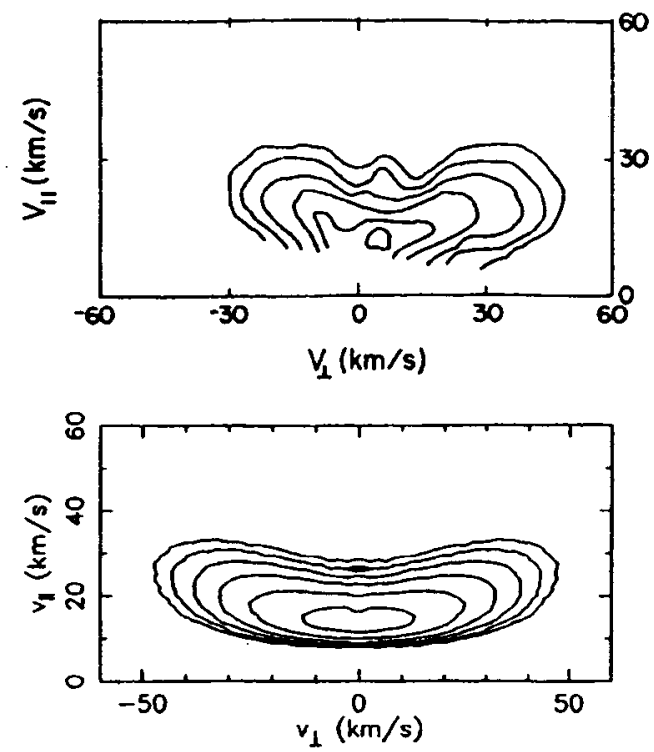

Figure 5-1. Observed and calculated velocity contour plots for conic event of Retterer et al. (1987).

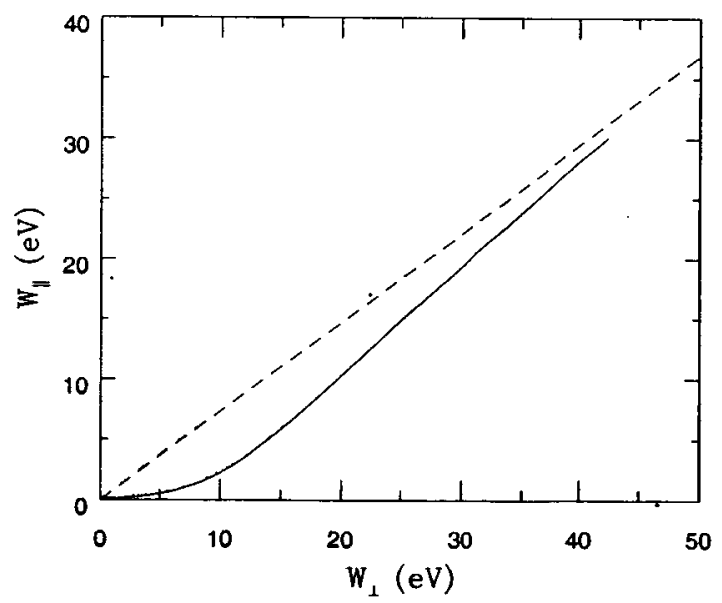

Figure 5-2. Solid line depicts $W_{\|}$versus $W_{\perp}$ for simulated conic events. Dashed line is the asymptote predicted by Chang et al. (1986). 
per oxygen ion as the ions evolve upward along the geomagnetic field line. We note that as the energies increase with altitudes, the ratio of the energies becomes nearly a constant.

These results are comparable to our previous calculated results based on the assumption that the relevant fluctuations were purely field-aligned propagating electromagnetic ion cyclotron waves (Chang et al., 1986; Retterer et al., 1987; Crew and Chang, 1988). As discussed in the previous sections, we generally expect the coexistence of non-propagating transverse electrostatic nonlinear fluctuations and a small fraction of field-aligned propagating waves in the auroral zone. Thus, the ion energization process in the auroral zone is probably due to a combination of both types of fluctuations. As it has been discussed in Chang et al. (1986), an asymptotic solution exhibiting such behavior may be obtained analytically in closed form. Therefore, in the asymptotic limit (i.e, at sufficiently high altitudes), it is expected that such an ion distribution will become entirely independent of its low altitude initial conditions. In fact, it has been shown by Crew and Chang (1988) that the ion distributions will become self-similar at sufficiently high altitudes and everything will scale with the altitude.

The above sample calculations did not include the self-consistent electric field that must be determined in conjunction with the energization of the ions as well as the electrons. This is particularly relevant in the downward auroral current region where the electric field can provide a significant pressure cooker effect such as that suggested by Gorney et al. (1985) and demonstrated convincingly by Jasperse (1998) and Jasperse and Grossbard (2000) based on global evolutional calculations similar to those considered by Tam and Chang $(1999 \mathrm{a}, \mathrm{b} ; 2001 ; 2002)$ for the solar wind and Tam et al. $(1995,1998)$ for the polar wind. These ideas will not be considered in this brief review.

We now return to the discussion of the effect of intermittency on ion heating. Measurements of the electric field spectral density are generally limited by the response capabilities of the measuring instruments. The faster the instruments can collect data, the more refined the scales of the measurements. As it has been seen in Section 4, we expect the measured spectrum density to exhibit small-scale intermittency behavior. In fact, it is known that fast response measurements generally exhibit strongly intermittent signatures of the fluctuations. In the diffusion approximation, the ion energization process is limited by the amplitude of the second moment of the probability distribution of the fluctuations. This amplitude may become smaller as the scale of measurements is reduced. Thus, in the limit of small scales, the amplitude of the measured spectrum may decrease and thereby requiring a larger value of $\eta$ to accomplish the same level of energization. 


\section{PLASMAS}

But, the effects of the intermittency of the fluctuations on particle energization may be underestimated if we stay, within the diffusion approximation. As it can be seen from the derivation of the diffusion approximation above, only the second order correlations of the fluctuations were included in the energization process. Since for intermittent turbulence, the probability distributions of the fluctuations are generally non-Gaussian, the effects of the intermittency can manifest in the higher order correlations beyond the second order diffusion coefficient. This implies that the higher order correlations of the velocity fluctuations may be of the order of $\Delta t$ and therefore cannot be neglected in (5.3). Under such circumstances, the Fokker-Planck and diffusion approximations of the ion energization processes can become inadequate. A more appropriate approach to address such non-Gaussian stochastic processes is to refer directly to the functional equation (5.2) using the non-Gaussian transition probability or the Langevin equation (5.1) with the actual intermittent time series of the electric field fluctuations. Again, the details of these ideas will not be considered in this review.

\section{Summary}

We have provided a modern description of dynamical complexity relevant to the intermittent turbulence of coexisting non-propagating spatiotemporal fluctuations and propagating modes in space plasmas. The theory is based on the physical concepts of sporadic and localized interactions of coherent structures that emerge naturally from plasma resonances.

The technique of the dynamic renormalization-group is applied to the study of forced and/or self-organized criticality (FSOC) and scale invariance related to such type of multiscale fluctuations. We also demonstrated that the particle interactions with the intermittent turbulence could lead to the efficient energization of the plasma populations such as auroral ions. Numerical examples are presented to illustrate the concepts and methodology.

\section{Acknowledgments}

This review touches upon a broad range of research areas covering the physics of space plasmas and complexity. The authors wish to thank their past and present colleagues, M. André, V. Angelopoulos, R. Bruno, S. 
Chandrasekhar, S.C. Chapman, G. Consolini, B. Coppi, G.B. Crew, G. Ganguli, M. Goldstein, A. Hankey, J.R. Jasperse, S. Jurac, C.F. Kennel, P. Kintner, M. Kivelson, A. Klimas, A.T.Y. Lui, E. Marsch, W. Matthaeus, P. De Michelis, J.F. Nicoll, J.M. Retterer, C. Seyler, A.S. Sharma, M.I. Sitnov, H.E. Stanley, D. Tetreault, V. Uritsky, J. Valdivia, D. Vassiliadis, D. Vvedensky, N. Watkins, J. Weygand, F. Yasseen, and J.E. Young for very useful discussions. Our wavelet analysis employed some of the wavelet software provided by $\mathrm{C}$. Torrence and $\mathrm{G}$. Compo, which is available at URL: http://paos.colorado.edu/research/wavelets. This research was partially supported by AFOSR, NASA and NSF.

\section{References}

André, M., and T. Chang, Ion heating perpendicular to the magnetic field, Physics of Space Plasmas, Scientific Publishers, Inc., eds.: T. Chang and J.R. Jasperse, Vol. 12, p. 35, 1992.

Angelopoulos, V., T. Mukai and S. Kokubun, Evidence for intermittency in Earth's plasma sheet and implications for self-organized criticality, Physics of Plasmas, 6, 4161, 1999.

Bak, P., C. Tang, and K. Wiesenfeld, Self-organized criticality: an explanation of $1 / \mathrm{f}$ noise, Phys. Rev. Lett., 59, 381, 1987.

Baker, D., A.J. Klimas, R.L. McPherron and J. Büchner, The evolution from weak to strong geomagnetic activity: An interpretation in terms of deterministic chaos, Geophys. Res. Lett., 17, 41, 1990.

Biskamp, D., Nonlinear Magnetohydrodynamics, Cambridge University Press, Cambridge, U.K., 1993.

Boffetta, G., V. Carbone, P. Giuliani, P. Veltri, and A. Vulpiani, Power laws in solar flares: Self-organized criticality or turbulence?, Phys. Rev. Lett. 83, 4662, 1999.

Bruno, R., V. Carbone, P. Veltri, E. Pietropaolo, B. Bavassano, Identifying intermittency events in the solar wind, Planetary and Space Science, 49, 1201, 2001.

Bruno, R., V. Carbone, L. Sorriso-Valvo, B. Bavassano, Truncated Levy-flight statistics recovered from interplanetary solar wind velocity and magnetic field fluctuations, EOS Trans. AGU, 83(47), Fall Meet. Suppl., Abstract SH12A-0396, 2002.

Castaing, B., Y. Cagne, and E.J. Hopfinger, Velocity probability density functions of high Reynolds number turbulence, Physica D., 46, 177, 1990.

Chandrasekhar, S., Stochastic problems in physics and astronomy, Rev. Mod. Phys., 15, 1, 1943.

Chang, T., and H.E. Stanley, Renormalization-group verification of crossover with respect to lattice anisotropy parameter, Phys. Rev., B8, 1178, 1973.

Chang, T., A. Hankey, and H.E. Stanley, Double-power scaling functions near tricritical points, Phys. Rev., B7, 4263, 1973a.

Chang, T., A. Hankey, and H.E. Stanley, Generalized scaling hypothesis in multi-component systems. I. Classification of critical points by order and scaling at tricritical points, Phys. Rev., B8, 346, 1973b.

Chang, T., J.F. Nicoll and J.E. Young, A closed-form differential renormalization-group generator for critical dynamics, Physics Letters, 67A, 287, 1978.

Chang, T., G.B. Crew, N. Hershkowitz, J.R. Jasperse, J.M. Retterer, and J.D. Winningham, Transverse acceleration of oxygen ions by electromagnetic ion cyclotron resonance with broadband left-hand polarized waves, Geophys. Res. Lett., 13, 636, 1986. 
PLASMAS

Chang, T., Low-dimensional behavior and symmetry breaking of stochastic systems near criticality - can these effects be observed in space and in the laboratory? IEEE Trans. on Plasma Science, 20, 691, 1992.

Chang, T., D.D. Vvedensky and J.F. Nicoll, Differential renormalization-group generators for static and dynamic critical phenomena, Physics Reports, 217, 279, 1992.

Chang, T., Sporadic, localized reconnections and multiscale intermittent turbulence in the magnetotail, AGU Monograph on "Encounter between Global Observations and Models in the ISTP Era", vol. 104, p.193, Horwitz, J.L., D.L. Gallagher, and W.K. Peterson, Am. Geophys. Union, Washington, D.C., 1998a.

Chang, T., Multiscale intermittent turbulence in the magnetotail, Proc. $4^{\text {th }}$ Intern. Conf. on Substorms, ed. Kamide, Y. et al., Kluwer Academic Publishers, Dordrecht and Terra Scientific Publishing Company, Tokyo, p. 431, 1998b.

Chang, T., Self-organized criticality, multi-fractal spectra, and intermittent merging of coherent structures in the magnetotail, Astrophysics and Space Science, ed. Büchner, J. et al., Kluwer Academic Publishers, Dordrecht, Netherlands, vol. 264, p. 303, $1998 \mathrm{c}$.

Chang, T., Self-organized criticality, multi-fractal spectra, sporadic localized reconnections and intermittent turbulence in the magnetotail, Physics of Plasmas, 6, 4137, 1999.

Chang, T., Colloid-like behavior and topological phase transitions in space plasmas: intermittent low frequency turbulence in the auroral zone, Physica Scripta, T89, 80, 2001.

Chang, T. and C.C. Wu, "Complexity" and anomalous transport in space plasmas, Physics of Plasmas, 9, 3679, 2002

Chang, T., C.C. Wu, and V. Angelopoulos, Preferential acceleration of coherent magnetic structures and bursty bulk flows in Earth's magnetotail, Physics Scripta, T98, 48, 2002.

Chang, T., "Complexity" induced plasma turbulence in coronal holes and the solar wind, in Solar Wind Ten, ed. by M. Velli, R. Bruno, and F. Malara, AIP Conference Proceedings, Vol. 679, Melville, NY, p. 481, 2003.

Chang, T., S.W.Y. Tam, C.C. Wu, and G. Consolini, Complexity, forced and/or selforganized criticality, and topological phase transitions in space plasmas, Space Science Reviews, 107, 425, 2003.

Chang, T., S.W.Y. Tam, and C.C. Wu, Complexity induced bimodal intermittent turbulence in space plasmas, Physics of Plasmas, to be published, 2004.

Chapman, S.C., N.W. Watkins, R.O. Dendy, P. Helander and G. Rowlands, Geophys. Res. Lett. 25, 2397, 1998.

Consolini, G., Sandpile cellular automata and magnetospheric dynamics, Cosmic Physics in the Year 2000, ed. S. Aiello, N. Lucci, G. Sironi, A. Treves and U. Villante, Soc. Ital. di Fis., Bologna, Italy, p. 123, 1997.

Consolini, G., and T. Chang, "Magnetic Field Topology and Criticality in Geotail Dynamics: Relevance to Substorm Phenomena", Space Science Reviews, 95, 309, 2001.

Consolini, G., T. Chang, and A.T.Y. Lui, Complexity and topological disorder in the Earth's magnetotail dynamics, to be published, 2004.

Crew, G.B., and T. Chang, Path-integral formulation of ion heating, Physics of Fluids, 31, $3425,1988$.

Einstein, A., Ann. d. Physik, 17, 549, 1905.

Farge, M., Wavelet transforms and their applications to turbulence, Annual Reviews of Fluid Mechanics, 24, 395, 1992.

Forman, M., and L.F. Burlaga, Exploring the Castaing distribution function to study intermittence in the solar wind at $\mathrm{LI}$ in June 2000 , in Solar Wind Ten, ed. M. Velli, R. Bruno, and F. Malara, AIP Conference Proceedings, Vol. 679, Melville, NY, p. 554, 2003. 
Freeman, M.P., N.W. Watkins and D.J. Riley, Evidence for a solar wind origin of the power law burst lifetime distribution of AE indices, Geophys. Res. Lett., 27, 1087, 2000.

Gomey, D.J., Y.T. Chiu, and D.R. Croley, Trapping of ion conics by downward parallel electric fields, J. Geophys. Res., 90, 4205, 1985.

Hnat, B., S.C. Chapman, G. Rowlands, N.W. Watkins, W.M. Farrel, Geophys. Res. Lett. 29(10), 10.1029/2001GL014587, 2002.

Hoshino, M., Nishida, A., Yamamoto, T., Kokubum, S., Turbulence magnetic field in the distant magnetotail: bottom-up process of plasmoid formation? Geophys. Res. Lett., 21, $2935,1994$.

Jasperse, J.R., Ion heating, electron acceleration, and self-consistent $E$ field in downward auroral current regions, Geophys. Res. Lett., 25, 3485, 1998.

Jasperse, J.R., and N.J. Grossbard, The Alfvén-Fälthammar formula for the parallel $E$-field and its analogue in downward auroral-current region, IEEE Trans. Plasma Science, 28, $1874,2000$.

Jurac, S., private communication, 2003.

Klimas, A.J., D.N. Baker, D.A. Roberts, D.H. Fairfield and J. Büchner, A nonlinear dynamical analogue model of geomagnetic activity, J. Geophys. Res., 97, 12253, 1992.

Klimas, A.J., J.A. Valdivia, D. Vassiliadis, D.N. Baker, M. Hesse, and J. Takalo, Selforganized criticality in the substorm phenomenon and its relation to localized reconnection in the magnetospheric plasma sheet, J. Geophys. Res., 105, 18765, 2000.

Lu, E.T., Avalanches in continuum driven dissipative systems, Phys. Rev. Lett., 74, 2511, 1995.

Lui, A.T.Y., Multiscale and intermittent nature of current disruption in the magnetotail, in Physics of Space Plasmas, MIT Geo/Cosmo Plasma Physics, eds.: T. Chang and J.R. Jasperse, vol. 15, p. 233, 1998.

Lui, A.T.Y., S.C. Chapman, K. Liou, P.T. Newell, C.I. Meng, M. Brittnacher, G.D. Parks, Is the dynamic magnetosphere an avalanching system?. Geophys. Res. Lett., 27, 911, 2000.

Matthaeus, W.H., and M.L. Goldstein, Low-frequency $1 / f$ noise in the interplanetary magnetic field, Phys. Rev. Lett., 57, 495, 1986.

Matthaeus, W.H., M.L. Goldstein and D.A. Roberts, Evidence for the presence of quasi-twodimensional nearly incompressible fluctuations in the solar wind, $J$. Geophys. Res., 95, $20673,1990$.

Meneveau, C., Analysis of turbulence in the orthogonal wavelet representation, J. Fluid Mech., 232, 469, 1991.

Milovanov, A., Zelenyi, L., Zimbardo, G., Fractal structures and power law spectra in the distant magnetotail, J. Geophys. Res., 101, 19903, 1996.

Morrison, P.J. and R.D. Hazeltine, Hamiltonian formulation of reduced magnetohydrodynamics, Phys. Fluids, 27, 886, 1984.

Nicoll, J.F., T. Chang, and H.E. Stanley, Nonlinear solutions of renormalization-group equations, Phys. Rev. Lett., 32, 1446, 1974.

Nicoll, J.F., T. Chang, and H.E. Stanley, Nonlinear crossover between critical and tricritical behavior, Phys. Rev. Lett., 36, 113, 1976.

Retterer, J.M., T. Chang, G.B. Crew, J.R. Jasperse and J.D. Winningham, Monte Carlo modeling of ionospheric oxygen acceleration by cyclotron resonance with broadband electromagnetic turbulence, Phys. Rev. Lett., 59, 148, 1987.

Rutherford, P.H., Nonlinear growth of the tearing mode, Phys. Fluids, 16, 1903, 1973.

Sharma, A.S., D. Vassiliadis and K. Papadopoulos, Reconstruction of low-dimensional magnetospheric dynamics by singular spectrum analysis, Geophys. Res. Lett., 20, 335, 1993. 
Seyler, C.E., Jr., Nonlinear 3-D evolution of bounded kinetic Alfvén waves due to shear flow and collisionless tearing instability, Geophys. Res. Lett., 15, 756, 1988.

Sitnov, M.I., A.S. Sharma, K. Papadopoulos, D. Vassiliadis, J.A. Valdivia, A.J. Klimas, and D.N. Baker, Phase transition-like behavior of the magnetosphere during substorms, $J$. Geophys. Res., 105, 12955, 2000.

Sorriso-Valvo, L., V. Carbone, P. Veltri, G. Consolini, and R. Bruno, Intermittency in the solar wind turbulence through probability distribution functions of fluctuations, Geophys. Res. Lett. 26, 1801, 1999.

Stanley, H.E., Introduction to Phase Transitions and Critical Phenomena, Oxford University Press, New York, 1971.

Strauss, H.R., Nonlinear, three-dimensional magnetohydrodynamics of noncircular tokamaks, Phys. Fluids, 19, 134, 1976.

Tam, S.W.Y., F. Yasseen, T. Chang, and S.B. Ganguli, Self-consistent kinetic photoelectron effects on the polar wind, Geophys. Res. Lett., 22, 2107, 1995.

Tam, S.W.Y., F. Yasseen, and T. Chang, Further development in theory/data closure of the photoelectron-driven polar wind and day-night transition of the outflow, Ann. Geophys., 16, 948, 1998.

Tam, S.W.Y. and T. Chang, Kinetic evolution and acceleration of the solar wind, Geophys. Res. Lett., 26, 3189, 1999a.

Tam, S.W.Y. and T. Chang, Solar wind acceleration, heating, and evolution with waveparticle interactions, Comments on Modern Physics, Vol. 1, Part C, 141, $1999 \mathrm{~b}$.

Tam, S.W.Y. and T. Chang, Effect of electron resonant heating on the kinetic evolution of the solar wind, Geophys. Res. Lett., 28, 11351, 2001.

Tam, S.W.Y. and T. Chang, Comparison of the effects of wave-particle interactions and the kinetic suprathermal electron population on the acceleration of the solar wind, Astronomy \& Astrophysics, 395, 1001, 2002.

Tetreault, D., Turbulent relaxation of magnetic fields, 1. Coarse-grained dissipation and reconnection, $J$. Geophys. Res., 97, 8531, 1992a; ibid, 2. self-organization and intermittency, 97, 8541, 1992b

Taylor, J.B., Relaxation of toroidal plasma and generation of reversed magnetic fields, Phys. Rev. Lett., 33, 1139, 1974.

Tu, C.Y. and E. Marsch, MHD structures, waves and turbulence in the solar wind: observations and theories, Space Science Reviews, 73, 1, 1995.

Uritsky, V.M., A.J. Klimas, D. Vassiliadis, D. Chua, and G.D. Parks, Scale-free statistics of spatiotemporal auroral emissions as depicted by POLAR UVI images: The dynamic magnetosphere is an avalanching system, J. Geophys. Res., 107, 1426, 2002.

Waddell, B.V., B. Carreras, H.R. Hicks, and J.A. Holmes, Nonlinear interaction of tearing modes in highly resistive tokamaks, Phys. Fluids, 22, 896, 1979.

Watkins, N. W., Scaling in the space climatology of the auroral indices: Is SOC the only possible description?, Nonlinear Processes in Geophysics, 9, 389, 2002.

Watkins, N.W., S.C. Chapman, R.O. Dendy and G. Rowlands, Robustness of collective behavior in strongly driven avalanche models: magnetospheric implications, Geophys. Res. Lett., 26, $2617,1999$.

Weygand, J., private communication, 2003.

Wilson, K.G. and J. Kogut, The renormalization group and the epsilon-expansion, Physics Reports, 12C, 76, 1974.

Wu, C.C., and T. Chang, 2D MHD Simulation of the Emergence and Merging of Coherent Structures, Geophys. Res. Lett., 27, 863, 2000a. 
Wu, C.C., and T. Chang, Dynamical evolution of coherent structures in intermittent twodimensional MHD turbulence, IEEE Trans. on Plasma Science, 28, 1938, $2000 \mathrm{~b}$.

Wu, C.C., and T. Chang, Further study of the dynamics of two-dimensional MHD coherent structures - A large scale simulation, Journal of Atmospheric Sciences and Terrestrial Physics, 63, 1447, 2001. 